have to be greater than $m_{v}+m_{\mu}$. Using the values of the above example, we should have $m_{\pi}>340 m_{e}>$ $m_{\mu}$, an inequality which does not seem contradictory to present experience. While on this assumption the $\sigma$-meson could not spontaneously give rise to a pair of $\mu$-mesons (charged and neutral), such a process would be possible in the field of a nucleus, if the energy is sufficient-as it might easily be under the Berkeley conditions. It is suggested that this may be the explana. tion of the comparatively large number of $\mu$-mesons observed in the Berkeley plates. While the most probable process under those conditions seems to be the formation of $\sigma-m e s o n s$ by Brems. strahlung, the observed cases of $\pi$. decay might on the above view be due to a break-up of nucleons, a process which would be expected to occur with measurable probability, the energy quantum (frequency multiplied by Planck's constant), corresponding to the collision time, being of the same order of magnitude as the assumed binding energy of the nucleon.

Without entering more closely on the consequences of the opposite assumptions-the identity of $\sigma$ - and $\pi$-mesons - for the above considera. tions, I should like to state that in my view the interpretation of the $\mu$-meson as an $\hbar / 2$-particle with an ordinary $\beta$-decay, which is, of course, independent of the hypothesis concerning nucleons and $\pi$-mesons, has not only a better foundation than the other assumptions of the above note, but also the more important bearing on the problem of the incorporation of meson theory in the general relativistic field theory. In this connexion it should perhaps be mentioned that the argument given in the note concerning the mass value of the electrophoton can only be expected to give the order of magnitude.

${ }^{1}$ Leprince-Ringuet, L., and L'Héritier, M., J. Phys. Radium, (8), 7, 66,69 (1946). Rochester, G. D., and Butler, C. C., Nature, 160, 855 (1947).

${ }^{2}$ Weisskopf, V., Phyః. Rev., 72, 155 (1947). Pontecorvo, B., Phys. Rev., 72, 246 (1947)

${ }^{3}$ Klein, O., Ark. and Mat. Astr. O. Fys., 34 A, No. 1 (1946).

- Konopinski, Rev. Mod. Phys., 15, 207 (1943).

\section{EXCITATION CURVES FOR THE $\gamma$-RADIATION FROM LIGHT ELEMENTS BOMBARDED WITH $a$-PARTICLES FROM POLONIUM}

\author{
By PROF. HILDING SLÄTIS \\ Nobel Institute for Physics, Stockholm
}

LARGE number of investigations have been
bombarded with $\alpha$-particles. In several cases excita.
tion curves have been obtained for the $\gamma$-radiation as
a function of the energy of the $\alpha$-particles. (A review
of earlier polonium $\alpha$-particle bombardments of light
elements, particularly those which may be of interest

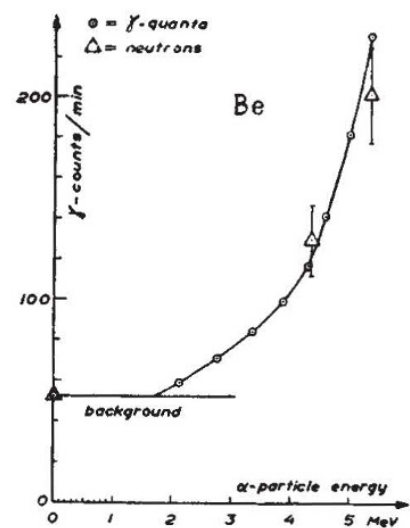

B
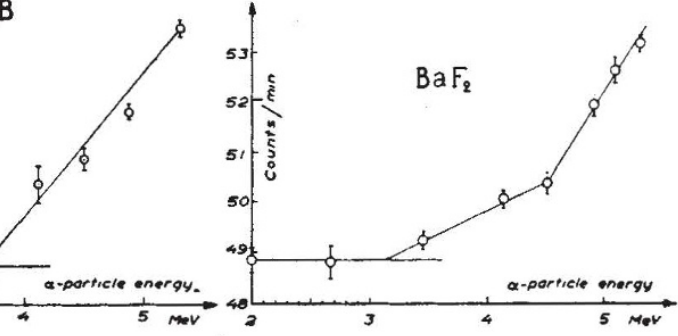

EMISSION OF $\gamma$-QUANTA $(\odot)$ FROM LITHIUM, BERYLLIUM, BORON,
AND NEUTRONS $(\triangle)$ FROM BERYLITUM $\triangle 8$ A PUNCTHON OF THE ENIERGY OF THE BOMBARDING $\alpha$-PARTICLES

for a possible $\gamma$-radiation, has been published by the present writer in Arkiv. Mat., Ast. Fysik, 35 A, No. 31 ; 1948). The results of different authors, however, are often inconsistent. For that reason I made use of an available polonium sample of about $40 \mathrm{mC}$. for observing the excitation functions of the $\gamma$-radiation from light elements bombarded with $\alpha$-particles. The energy of the $\alpha$-particles was varied by means of carbon dioxide.

Lithium, beryllium, boron, carbon, nitrogen, oxygen, fluorine, sodium, magnesium, aluminium, silicon, phosphorus, sulphur, chlorine, potassium, calcium and lead were investigated. $\gamma$-Emission was observed from lithium, beryllium, boron, nitrogen, fluorine, sodium, magnesium and aluminium. The intensity of the $\gamma$-radiation was measured as a function of the energy of the $\alpha$-particles for all these except nitrogen (Figs. 1-2). The $\alpha$-particle threshold values for $\gamma$-emission and the relative intensities (as percentage of the measured polonium $\gamma$-radiation background) of the $\gamma$-quanta are given in the accompanying table.

\begin{tabular}{|l|c|c|}
\hline Element & $\begin{array}{c}\gamma \text {-Emission starts at } \\
\text { the } \alpha \text {-energy }\end{array}$ & $\begin{array}{c}\text { Intensity of the } \gamma \text {-radia- } \\
\text { tion for } 5 \cdot 3 \mathrm{MeV}, \alpha \text {-particles }\end{array}$ \\
\hline Li & MeV. & \\
Be & $2 \cdot 3$ & 61 \\
B & $1 \cdot 7$ & 340 \\
N & $3 \cdot 7$ & $9 \cdot 0$ \\
Fa & $3 \cdot 1$ (and $4 \cdot 5)$ & $2 \cdot 7$ \\
Mg & $4 \cdot 6$ & $9 \cdot 0$ \\
Al & $3 \cdot 6$ & $9 \cdot 5$ \\
& $4 \cdot 1$ & $3 \cdot 7$ \\
\hline
\end{tabular}

The statistical errors are represented in all the diagrams except the $\gamma$-emission curves for lithium and beryllium, where the errors were too small to be 


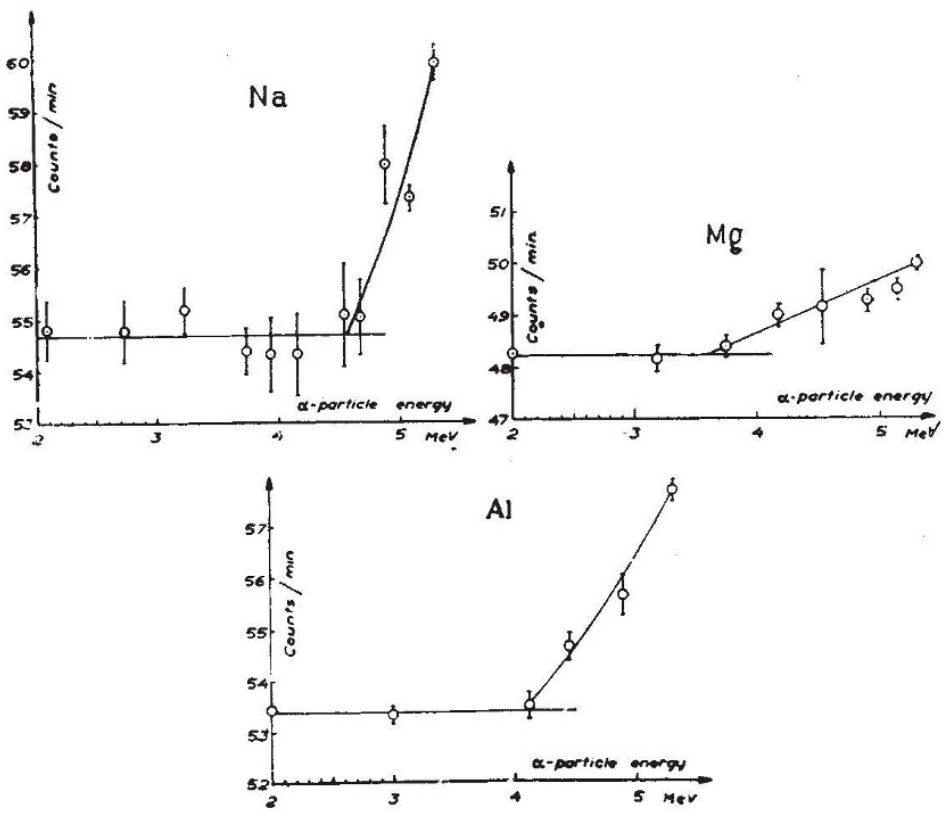

Fig. 2. $\gamma$-EMISSION FROM SODIUM, MAGNESIUM AND ALUMINIUM AS A FUNCTION OF THE ENERGY OF THE BOMBARDING $\alpha$-PARTICLES

plotted $(\lesssim 1$ per cent of the polonium $\gamma$-radiation background).

The neutron-emission from beryllium was measured for $\alpha$-particles of energies $4 \cdot 3$ and $5 \cdot 3 \mathrm{MeV}$. The results and the statistical errors are plotted on an arbitrary scale in Fig. 1.

${ }_{8} \mathbf{L i}$. The $\gamma$-radiation is due to an excitation of the $\mathrm{Li}^{7}$-nucleus by inelastic collisions with $\alpha$-particles, as quoted, for example, in the paper by Siegbahn and Slätis ${ }^{1}$. The $\gamma$-radiation starts for $\alpha$-particles of the energy $2 \cdot 3 \mathrm{MeV}$., in agreement with the result of Schnetzler ${ }^{2}$ but not with earlier investigations by Webster $^{3}(1.9 \mathrm{MeV}$.$) , Curie and Joliot { }^{4}(3 \cdot 1)$, Savel ${ }^{5}$ $(2 \cdot 8-3 \cdot 0)$.

${ }_{4}$ Be. The $\gamma$-radiation maximum with $2 \cdot 7 \mathrm{MeV}$. $\alpha$-particles found by Becker and Bothe ${ }^{6}$ could not be confirmed, nor the starting point of the $\gamma$-emission for $\alpha$-particles of the range $1.7 \mathrm{~cm}$. (3 MeV.). The neutrons seem to be emitted simultaneously with the $\gamma$-quanta, as predicted by Chadwick? ${ }^{7}$ The energy (1.7 MeV.) of the $\alpha$-particles at which the $\gamma$-emission. starts is the same as Chadwick found for the beginning of the neutron emission, and is in fairly good agreement with the starting points for the $\gamma$-emission found by Webster ${ }^{3}(1.9 \mathrm{MeV}$.) and by Szalay and Zimonyi $^{8}\left(1.62 \mathrm{MeV}\right.$.). Rasetti ${ }^{9}$ found $1.6 \mathrm{MeV}$. for the beginning of neutron emission and Curie and Joliot ${ }^{4} 1.3 \mathrm{MeV}$. for neutrons and $\gamma$-quanta.

${ }_{5}$ B. According to Becker and Bothe ${ }^{6}$, the $\gamma$-radiation begins at the same $\alpha$-particle energy for which the second proton group starts (also in agreement with v. Baeyer $\left.{ }^{10}\right)$. The corresponding $\alpha$-particle range was $2.90 \mathrm{~cm}$. $(4.4 \mathrm{MeV}$.). This is not in agreement with the curve in Fig. 1, which starts at 3.7 MeV., or with the results of Webster ${ }^{3}(1.9 \mathrm{MeV}$.), or those of Szalay and Zimonyi $(1 \cdot 5 \mathrm{~cm}$. $=2 \cdot 7 \mathrm{MeV}$.), but in good accordance with Heidenreich ${ }^{11}(3.6 \mathrm{MeV}$.) and Alichanow, Alichanian and Dzelepow ${ }^{12}(2 \cdot 1 \mathrm{~cm}$. = $3.5 \mathrm{MeV}$.).

${ }_{8}$ F. The first proton pair found by Chadwick and Constable ${ }^{13}$ started at an $\alpha$-range of $2 \cdot 2 \mathrm{~cm}$. $(3 \cdot 64 \mathrm{MeV}$.), the second pair at $2 \cdot 7 \mathrm{~cm}$. $(4 \cdot 2 \mathrm{MeV}$.).
This result is not in agreement with my observations; I find that (Fig. 1) the $\gamma$-radiation starts at $3 \cdot 1 \mathrm{MeV}$. with clearly a second component at $4.5 \mathrm{MeV}$. energy of the $\alpha$-particles. The starting point $0 \cdot 9-1 \cdot 2 \mathrm{MeV}$., found by Savel $^{5}$, seems to be very improbable. Speh ${ }^{14}$ discovered that the $\gamma$ radiation consisted of two components. The harder one started at $3.1 \mathrm{~cm} . \alpha$ particle range $(4.6 \mathrm{MeV}$.), which is in good agreement with the second starting point of the $\gamma$-radiation shown in Fig. 1.

${ }_{11} \mathrm{Na}$. According to Fig. 2, $\gamma$-emission starts at an $\alpha$-particle energy of $4 \cdot 6$ $\mathrm{MeV}$. This agrees neither with Savel's ${ }^{5}$ result (3.7-4 MeV.) nor with Kovács's ${ }^{15}$ $\left(2 \cdot 7 \mathrm{MeV}\right.$. and $3.5 \mathrm{MeV}$.). König ${ }^{16}$, however, found a proton resonance group, called $B$, which started at 4.8 MeV. This is in accordance with my result.

${ }_{12}$ Mg. Klarmann's ${ }^{17}$ first proton group started at an $\alpha$-particle energy of $3 \cdot 26 \mathrm{MeV}$, which may agree with my value $3.6 \mathrm{MeV}$. for the beginning of the $\gamma$-radiation (Fig. 2). Savel $^{5}$ found 4.3-4.5 MeV.

${ }_{13}$ Al. Pose's ${ }^{18}$ first proton group for an $\alpha$-range of $2.50 \mathrm{~cm}$. (4 MeV.) agrees well with the $\alpha$-particle threshold value $4 \cdot 1 \mathrm{MeV}$. for the $\gamma$-emission found by $m \theta$ (Fig. 2). Chadwick and Constable ${ }^{13}$ found for the first proton pair a threshold value $4 \cdot 2 \mathrm{MeV}$. for the $\alpha$-particle energy, also in good accordance with my result. Savel ${ }^{5}$ found $3-3 \cdot 3 \mathrm{MeV}$. for the beginning of the $\gamma$-emission, Fahlenbrach ${ }^{19} 3 \mathrm{~cm}$. $(4 \cdot 5 \mathrm{MeV}$.) for the starting point of the $(\alpha, p)$-process, Alichanow, Alichanian and Dzelepow ${ }^{12} 3 \cdot 4 \mathrm{~cm} .(4 \cdot 9 \mathrm{MeV}$.) for the $(\alpha, n)$-process. For the same $(\alpha, n)$-process Waring and Chang ${ }^{20}$ obtained $3 \cdot 4 \mathrm{MeV}$. Pollard, Schultz and Brubaker ${ }^{21}$ found possible resonance effects at $2 \cdot 3 \mathrm{~cm}$. $(3.8 \mathrm{MeV}$.) and $4.8 \mathrm{~cm}$. (6 MeV.). The former $\alpha$-particle energy $3.8 \mathrm{MeV}$,, as well as Fünfer's ${ }^{22}$ value $2.3 \mathrm{~cm}$. $(3.8 \mathrm{MeV}$.) and the starting point $2.4 \mathrm{~cm}$. $(3.9 \mathrm{MeV}$.) for the $\gamma$-radiation, found by Szalay and Zimonyi ${ }^{8}$, agree well with my $\alpha$-particle energy value $4.1 \mathrm{MeV}$. for the beginning of the $\gamma$-radiation from aluminium.

${ }^{2}$ Siegbahn, K., and Slatis, H., Nature, 159, 471 (1947) ; Arkiv. Mat., Ast. Fysik, 34 A, No. 15 (1947).

' Sehnetzler, K., Z. Phys., 95, 302 (1935).

${ }^{3}$ Webster, H. C., Proc. Roy. Soc., A, 136, 428 (1932).

4 Curie, J., and Joliot, F., C.R. Acad. Sci., Paris, 196, 397 (1933).

${ }^{5}$ Savel, P., Ann. de Phys., 4, 88 (1935).

- Becker, H., and Bothe, W., Naturwiss., 20, 349 (1932).

'Chadwick, J., Proc. Roy. Soc., A, 142, 1 (1933).

${ }^{8}$ Szalay, A., and Zimonyi, J., Z. Phys., 115, 639 (1940).

- Rasetti, $Z$. Phys., 78, 165 (1932).

${ }^{19}$ v. Baeyer, H. J., Z. Phys., 95, 417 (1935).

"Heidenreich, F., Z. Phys., 86, 675 (1938).

${ }^{12}$ Alichanow, A. I., Alichanian, A. I., and Dzelepow, B. S., Z. Phys., 98, 350 (1935).

${ }^{13}$ Chadwick, J., and Constable, J. E. R., Proc. Roy. Soc., A, 135, 48 (1932).

14 Speh, K. C., Phys, Rev., 50, 689 (1936).

${ }^{15}$ Kováes, M., Phys. Rev., '70, 895 (1946).

${ }^{16}$ König, A., Z. Phys., 90, 197 (1934); Naturwis8., 22, 150 (1934),

${ }^{17}$ Klarmann, H., Z. Phys., 87, 411 (1934).

18 Pose, H., Phys. Z., 30, 780 (1929) ; Naturwiss., 17, 624 (1929).

${ }^{19}$ Fahlenbrach, H., Z. Phys., 94, 607 (1935).

${ }^{20}$ Waring, I. R. S., and Chang, W. Y., Proc. Roy. Soc., A, 157, 652 (1936).

${ }^{21}$ Pollard, E., Schultz, H. I., and Brubaker, G., Phys. Rev., 53, 351 (1938).

22 Fünfer, E., Ann. de Phys., 32, 313 (1938). 